\title{
Models of boundary behavior of particles diffusing between two concentrations
}

\author{
Amit Singer $^{a}$, Zeev Schuss ${ }^{a}$, Boaz Nadler ${ }^{b}$ and Robert S. Eisenberg ${ }^{c}$ \\ ${ }^{a}$ Department of Applied Mathematics, Tel-Aviv University, \\ Ramat-Aviv, Tel-Aviv 69978, Israel; \\ ${ }^{b}$ Department of Mathematics, Yale University, \\ 10 Hillhouse Ave., P.O. Box 208283, New Haven, CT 06520-8283; \\ ${ }^{c}$ Department of Molecular Biophysics and Physiology, Rush Medical Center, \\ 1750 Harrison St., Chicago, IL 60612*
}

\begin{abstract}
Flux between regions of different concentration occurs in nearly every device involving diffusion, whether an electrochemical cell, a bipolar transistor, or a protein channel in a biological membrane. Diffusion theory has calculated that flux since the time of Fick (1855), and the flux has been known to arise from the stochastic behavior of Brownian trajectories since the time of Einstein (1905), yet the mathematical description of the behavior of trajectories corresponding to different types of boundaries is not complete. We consider the trajectories of non-interacting particles diffusing in a finite region connecting two baths of fixed concentrations. Inside the region, the trajectories of diffusing particles are governed by the Langevin equation. At the interface between the region and the baths, trajectories are set by a control mechanism that modifies dynamics so the concentration of particles remains (nearly) constant. We analyze different models of controllers and derive equations for the time evolution and spatial distribution of particles inside the domain. Our analysis shows a distinct difference between the time evolution and the steady state concentrations. While the time evolution of the density is governed by an integral operator, the spatial distribution is governed by the familiar Fokker-Planck operator. The boundary conditions for the time dependent density depend on the model of the controller; however, this dependence disappears in the steady state, if the controller is of a renewal type. Renewal-type controllers, however, produce spurious boundary layers that can be catastrophic in simulations of charged particles, because even a tiny net charge can have global effects. The design of a non-renewal controller that maintains concentrations of non-interacting particles without creating spurious boundary layers at the interface requires the solution of the time-dependent Fokker-Planck equation with absorption of outgoing trajectories and a source of ingoing trajectories on the boundary (the so called albedo problem).
\end{abstract}

Keywords Non-equilibrium diffusion, ion channels, boundary conditions

\section{INTRODUCTION}

We consider particles that diffuse between two regions where average concentrations are maintained at constant unequal values (see fig. 1). Flux between regions of different concentration occurs in nearly every device involving diffusion, whether an electrochemical cell, a bipolar transistor, or a protein channel in a biological membrane. Continuum theories of such diffusive systems describe the concentration field by the (time independent) NernstPlanck equation with fixed boundary concentrations. ${ }^{1-7}$

The microscopic theory underlying diffusion describes motion of particles by Langevin's equations ${ }^{3,5,8-10}$ everywhere, except at the boundaries. The behavior of the Langevin trajectories at the boundaries depends on the interaction between the particles and the boundaries. Thus, for example, outgoing trajectories can be terminated (absorbed); reflected (or otherwise reinjected); delayed; and so on. None of this is described by the

Further author information: E-mail addresses and tel.: A.S.: amit_sin@zahav.net.il, Z.S.: schuss@post.tau.ac.il, Telephone: +972 (3) 640 8827, B.N.: boaz.nadler@yale.edu, Telephone: 1203432 4172, R.S.E: beisenbe@rush.edu, Telephone: 13129426467 , Send correspondence to A.S. 


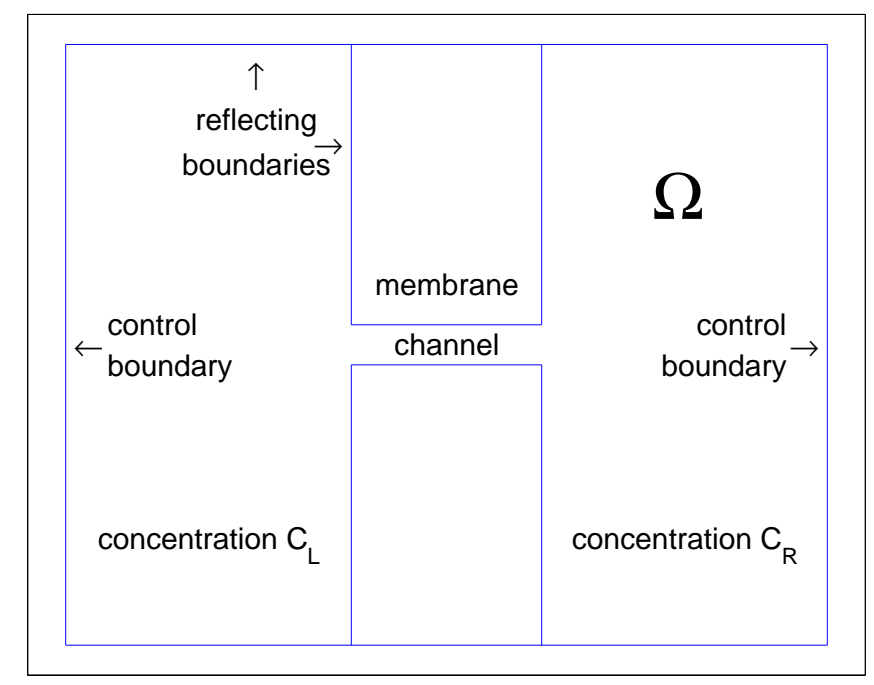

Figure 1. The concentration cell of experimental electrochemistry and molecular biophysics. The region $\Omega$ typically consists of small parts of two large baths of effectively constant concentrations, separated by a permeable membrane in experimental electrochemistry, or (in biophysics) an impermeable membrane containing one or more channels.

Langevin equations. Brownian dynamics cannot describe such boundary behavior, because Brownian particles have no definite velocity, being functions of infinite variation. Particles with positive (e.g., incoming) velocities can be distinguished from those with negative (e.g., outgoing) velocities, only if particles have a definite velocity. ${ }^{8}$ The Langevin equations are often directly integrated in simulations. ${ }^{11-21}$

In devices, the interaction between the trajectories and the boundaries must be specified because the inputs, outputs, and power supplies of devices are at their boundaries; in physical systems, the boundaries are where charge, matter, and energy are injected into a device; in biological systems boundaries represent reservoirs maintained at a (nearly) fixed electrochemical potential by active processes of the cell.

The formulation of boundary conditions for the particle concentration is obvious in macroscopic models, but formulation of boundary conditions for the underlying trajectories is not so clear cut, particularly because many different physical or computational control mechanisms can maintain a constant average density at prescribed locations, usually near the boundaries. ${ }^{11-15,19,22-28}$ Many boundary conditions used in Brownian and Langevin simulations produce spurious boundary layers, that do not exist at those locations in the physical systems being simulated. Spurious boundary layers are particularly damaging to simulations of charged particles. A boundary layer leads to large fluctuations in the electrostatic field which spreads over the entire simulation region. This was clearly demonstrated in Ref 16 for a problem with equal boundary concentrations in a simulation with a buffer zone.

In this paper we provide a general description of the concentration and flux of non-interacting particles diffusing between constant concentrations near the boundaries. We study renewal-type controllers that maintain fixed concentrations near the boundaries, determining the time course both of concentration (in phase space) and current. We show that the concentration is a weighted sum of "left" and "right" concentrations, each of which satisfies a different integro-partial-differential equation and different boundary conditions. In the steady state the phase space concentration is the weighted sum of the solutions of two stationary solutions of the so called albedo problem. ${ }^{29-34}$ The albedo problem was first posed by Wang and Uhlenbeck ${ }^{35}$ in 1945 and its analytic solution was first found by Marshall and Watson. ${ }^{36}$ Further progress was made by Hagan, Doering, and Levermore, ${ }^{37,38}$ who used complex analysis to solve the half range expansion problem. The solution employed here was found by Kłosek. ${ }^{39}$ The weights in the sum of "left" and "right" concentrations are the rates at which the controllers inject trajectories into the system. 
Different control mechanisms that maintain the same concentrations near the boundaries produce different time operators that govern the evolution of the "left" and "right" concentrations. Each evolution is nonMarkovian. The removal and injection - or re-injection - of particles into the system by renewal-type boundary controllers are described by renewal-type integral operators that govern the time evolutions of these concentrations, in contrast to the Fokker-Planck or Nernst-Planck equations that are commonly used. ${ }^{5,40,41}$

The description of this simplified model of diffusion of non-interacting particles is apparently new: we include a detailed description of the physical mechanism that maintains the non-equilibrium state of the system. Similar descriptions are needed when particles interact.

An alternative approach (see Section 4) is to assume that trajectories are reflected at boundaries, so that reflecting boundary conditions are imposed on the solution of the Fokker-Planck or Nernst-Planck equations. The (quasi) steady state is achieved when all transient modes have decayed except the first one. This method was used in Refs 42,43 for non-interacting particles, and we think it can also be used to describe systems of interacting particles.

\section{FORMULATION}

We consider a system composed of two finite macroscopic volumes containing electrolyte solutions of different ionic species, connected by a macroscopic or microscopic channel. A control mechanism keeps different average concentrations in the two volumes, so that a steady current flows through the system (see fig. 1), thus keeping it out of equilibrium. As seen in the figure, the control mechanism is located only on parts of the boundaries of the system, at macroscopic distances away from the connecting channel. The control mechanism re-injects exiting trajectories at one or the other boundaries in a way that maintains average fixed concentrations near the boundaries at all times. We have in mind, for example, a typical setup used to measure the diffusion of ions through a protein channel of a biological cell membrane that separates two solutions of different fixed concentrations. ${ }^{1}$ Alternatively, all trajectories are reflected at the boundary so that the system reaches equilibrium after a long time, but the long lasting transient regime is the non-equilibrium regime in which an almost steady current flows between the baths. This time behavior occurs when the number of particles that flow through the channel during the period of measurement is much smaller than the total number of ions in either bath.

The problem at hand is to describe the steady diffusion current flowing between the two baths, in terms of the molecular properties of the diffusing ions, such as their radii and interaction forces, as a function of the experimentally controlled variables, such as the concentrations in the two baths and the external potential, and as a function of the system geometry, e.g., the geometry and charge distribution of the channel.

The particles diffuse in a domain $\Omega$ that consists of the two macroscopic volumes and the connecting channel. We assume that there are $N^{h}$ ions of species $h\left(h=\mathrm{Ca}^{++}, \mathrm{Na}^{+}, \mathrm{Cl}^{-}, \ldots\right)$ in $\Omega$, which are numbered at time $t=0, \sum_{h} N^{h}=N$, and we follow their trajectories, $\boldsymbol{x}_{j}^{h}(t)=\left(x_{j}^{h}(t), y_{j}^{h}(t), z_{j}^{h}(t)\right)$ at all times $t>0\left(\boldsymbol{x}_{j}^{h}(t)\right.$ is the location of the $j$-th ion of species $h$ at time $t$ ).

For future use, the coordinate and velocity vectors of all ions in the $3 N$-dimensional configuration space, are denoted by $\tilde{\boldsymbol{x}}=\left(\boldsymbol{x}_{1}^{h_{1}}, \ldots, \boldsymbol{x}_{N^{h_{1}}}^{h_{1}}, \boldsymbol{x}_{1}^{h_{2}}, \ldots, \boldsymbol{x}_{N^{h_{2}}}^{h_{2}}, \ldots\right)$ and $\dot{\tilde{\boldsymbol{x}}}$ or $\tilde{\boldsymbol{v}}$.

\subsection{Equations of motion}

As in Ref 5, we assume that the motion of an ion in the solution is overdamped diffusion in a field of force. The source of the noise and friction is the thermal motion of the solvent (e.g., water) and both are interrelated by Einstein's fluctuation-dissipation principle. ${ }^{3}$ More specifically, our starting point is a memoryless system of $N$ coupled Langevin equations for the dynamics of all particles of the different species $h=\mathrm{Ca}^{++}, \mathrm{Na}^{+}, \mathrm{Cl}^{-}, \ldots$,

$$
\ddot{\boldsymbol{x}}_{j}^{h}+\gamma^{h}\left(\boldsymbol{x}_{j}^{h}\right) \dot{\boldsymbol{x}}_{j}^{h}=\frac{\boldsymbol{f}_{j}^{h}(\tilde{\boldsymbol{x}})}{M^{h}}+\sqrt{\frac{2 \gamma^{h}\left(\boldsymbol{x}_{j}^{h}\right) k_{B} T}{M^{h}}} \dot{\boldsymbol{w}}_{j}^{h}, \quad\left(j=1,2, \ldots, N^{h}\right),
$$

where a dot on top of a variable means differentiation with respect to time, $\gamma^{h}\left(\boldsymbol{x}^{h}\right)$ is the location dependent friction coefficient per unit mass and $M^{h}$ is the effective mass of an ion of species $h$. The force $\boldsymbol{f}_{j}^{h}(\tilde{\boldsymbol{x}})$ on the $j$-th 
ion of species $h$ includes all ion-ion interactions and thus depends on the locations of all ions. The functions $\dot{\boldsymbol{w}}_{j}^{h}$ are, by assumption, independent standard Gaussian white noises. The parameter $k_{B}$ is Boltzmann's constant and $T$ is absolute temperature. As seen in fig. 1, some parts of the boundary $\partial \Omega$ are reflecting, while other parts contain the control mechanism. At the boundary $\partial \Omega$, the random trajectories of the Langevin equations $(1)$ are either reflected or are redirected by the external control mechanism.

\section{RENEWAL CONTROLS}

The solution of (1) depends on the specific choice of control mechanism. We first analyze controls for onedimensional non-interacting systems because the treatment of three-dimensional interacting particle systems is more complicated. In this section we show that renewal controls (to be defined in subsection 3.3) reproduce correct macroscopic properties such as total net flux and concentration profile, but also produce non-physical boundary layers for non-interacting diffusive particle systems.

Consider particles diffusing in the interval $\Omega=[0, d]$. The control mechanism maintains average concentrations $C_{L}$ and $C_{R}$ at $0 \ll x_{L}<x_{R} \ll d$, respectively, away from the boundaries, where concentrations are actually measured. Each particle satisfies a Langevin equation

$$
\ddot{x}+\gamma \dot{x}+U^{\prime}(x)=\sqrt{2 \gamma \epsilon} \dot{w} .
$$

In order to complete the description of the dynamics we have to describe the motion of particles at the boundaries, i.e., to describe the action of the control mechanism.

\subsection{Probabilistic Control}

A possible control mechanism operates as follows: when a particle reaches either one of the boundaries, it tosses a Bernoulli coin with probabilities $(L, R), L+R=1, L, R \geq 0$. The control mechanism decides to re-enter the particle at the left boundary $x=0$ with probability $L$, and to re-inject the particle to the bath at the right boundary $x=d$ with probability $R$. The re-injections occur at random times; a particle that reached the boundary at time $t$, is delayed in the boundary a random time $T$ and re-injected at time $t+T$. The random time $T$ is a non-negative random variable with PDF

$$
q(s) d s=\operatorname{Pr}\{s \leq T<s+d s\} .
$$

The velocity of injection is distributed according to pre-determined distributions $s_{L}(v)$ and $s_{R}(v)$ of the left and right sources, respectively. For example, if both sources are Maxwellian, then

$$
s_{L}(v)=\frac{2}{\sqrt{2 \pi \epsilon}} e^{-v^{2} / 2 \epsilon}=s_{R}(-v), \quad v>0
$$

As shown below, the precise velocity distribution of the sources is unimportant for measurement of concentrations away from the boundaries.

The dynamics (1) and the boundary behavior provide a complete description of the trajectories, and therefore determine the probability distribution of the random particle trajectories in the system at any time. Assuming, as we may, that the precise velocity distributions is unimportant, there are only two parameters to be determined, namely the fixed number of particles in the system $N$ and the re-injection probability $R$. These two parameters determine uniquely the two measured concentrations $C_{L}$ and $C_{R}$.

Let $p_{i}(x, v, t)$ be the probability of finding the $i-t h$ particle at location $x$ and velocity $v$ at time $t$, given that it was injected to the bath at time $t=0$, from either the left or right boundary with probabilities $R$ and $L$, and the corresponding velocity distributions $s_{L}$ and $s_{R}$. Since the particles are independent and interchangeable, we find that $p_{1}=p_{2}=\ldots=p_{N}$, and set $p(x, v, t)=p_{1}(x, v, t)$. Let $p(x, v)$ be the steady state density of a single particle, i.e., $p(x, v)=\lim _{t \rightarrow \infty} p(x, v, t)$. The steady state concentration at location $x$ is given by

$$
C(x)=N \int_{-\infty}^{\infty} p(x, v) d v
$$


We use renewal theory ${ }^{44}$ to calculate $p(x, v)$. Suppose the device was turned on at time $t=0$. Let $t_{0}$ be the first time that the particle was injected into the system. Then the probability of finding the particle in location $(x, v)$ of the phase space at time $t$ is given by

$$
p(x, v, t)=\int_{0}^{t} p\left(x, v, t \mid t_{0}=s\right) q(s) d s .
$$

Let $\tau_{1}$ be the first passage time of the particle to the boundary. Conditioning on $\tau_{1}$ yields

$$
p(x, v, t)=\int_{0}^{t} q(s) d s \int_{0}^{\infty} p\left(x, v, t \mid t_{0}=s, \tau_{1}=r\right) p\left(\tau_{1}=r \mid t_{0}=s\right) d r
$$

where $p\left(\tau_{1}=r \mid t_{0}=s\right)=p(\tau=r-s)=0$ for $r<s$. We separate the integral into two parts

$$
\begin{aligned}
p(x, v, t)= & \int_{0}^{t} q(s) d s \int_{0}^{t} p\left(x, v, t \mid t_{0}=s, \tau_{1}=r\right) p(\tau=r-s) d r \\
& +\int_{0}^{t} q(s) d s \int_{t}^{\infty} p\left(x, v, t \mid t_{0}=s, \tau_{1}=r\right) p(\tau=r-s) d r \\
= & \int_{0}^{t} q(s) d s \int_{0}^{t} p(x, v, t-r) p(\tau=r-s) d r+f(x, v, t)
\end{aligned}
$$

where

$$
f(x, v, t)=\int_{0}^{t} q(s) d s \int_{t}^{\infty} p\left(x, v, t \mid t_{0}=s, \tau_{1}=r\right) p(\tau=r-s) d r .
$$

Changing the order of integration in (8) we obtain

$$
\begin{aligned}
p(x, v, t) & =\int_{0}^{t} p(x, v, t-r) d r \int_{0}^{t} q(s) p(\tau=r-s) d s+f(x, v, t) \\
& =\int_{0}^{t} p(x, v, t-r)\left(p_{\tau} * q\right)(r) d r+f(x, v, t)=\left(p * p_{\tau} * q\right)(t)+f(x, v, t),
\end{aligned}
$$

where $*$ denotes convolution. Taking the Laplace transform of the equation gives

$$
\hat{p}(x, v, \theta)=\frac{\hat{f}(x, v, \theta)}{1-\hat{p}_{\tau}(\theta) \hat{q}(\theta)} .
$$

The steady state distribution is given by

$$
p(x, v)=\lim _{t \rightarrow \infty} p(x, v, t)=\lim _{\theta \rightarrow 0} \theta \hat{p}(x, v, \theta)=\lim _{\theta \rightarrow 0} \frac{\theta \hat{f}(x, v, \theta)}{1-\hat{p}_{\tau}(\theta) \hat{q}(\theta)} .
$$

Both numerator and denominator of the right hand side vanish as $\theta$ tends to 0 . Expanding the denominator in Taylor series, we find that

$$
p(x, v)=\frac{\hat{f}(x, v, \theta=0)}{E \tau+E T},
$$

where $E \tau$ is the mean first passage time (MFPT), and $E T$ is the mean delay time before re-injection.

To evaluate $\hat{f}(x, v, \theta=0)$, we consider a Langevin particle in the interval $[0, d]$ which is injected at time $t=0$ at $x=0$ with velocity distribution $s_{L}(v)$. When the particle reaches one of the boundaries, it is absorbed, and its trajectory is terminated at once. Let $\tilde{p}_{L}(x, v, t)$ be the probability density function of the particle $(p$ should not to be confused with $\tilde{p}_{L}$; the subscript $L$ stands for left.) The density $\tilde{p}_{L}$ satisfies the Fokker-Planck equation

$$
\frac{\partial \tilde{p}_{L}}{\partial t}=\mathcal{L}_{x, v} \tilde{p}_{L}=-v \frac{\partial \tilde{p}_{L}}{\partial x}+\frac{\partial}{\partial v}\left[\left(\gamma v+U^{\prime}(x)\right) \tilde{p}_{L}\right]+\epsilon \gamma \frac{\partial^{2} \tilde{p}_{L}}{\partial v^{2}},
$$


with the initial condition

$$
\tilde{p}_{L}(x, v, t=0)=\delta\left(x-0^{+}\right) s_{L}(v),
$$

and the absorbing boundary conditions

$$
\begin{aligned}
\tilde{p}_{L}\left(x=0^{-}, v, t\right) & =0, v>0, \\
\tilde{p}_{L}(x=d, v, t) & =0, v<0 .
\end{aligned}
$$

Equations (14)-(17) define the time dependent albedo problem. In the limit of high friction a new time scale is often used ${ }^{10}$

$$
\hat{t}=t / \gamma
$$

so eq.(14) is rewritten as

$$
\frac{1}{\gamma} \frac{\partial \tilde{p}_{L}}{\partial \hat{t}}=\mathcal{L}_{x, v} \tilde{p}_{L}
$$

We define the function

$$
P_{L}(x, v)=\int_{0}^{\infty} \tilde{p}_{L}(x, v, \hat{t}) d \hat{t}=\frac{1}{\gamma} \int_{0}^{\infty} \tilde{p}_{L}(x, v, t) d t .
$$

The function $\gamma P_{L}(x, v)$ is the average time that a particle spends at location $(x, v)$ prior to its absorption, given that it was injected from the left electrode at time $t=0$. It follows from equations (14)-(17) that $P_{L}$, the solution of the steady state albedo problem, satisfies

$$
\mathcal{L}_{x, v} P_{L}=-\frac{1}{\gamma} \delta\left(x-0^{+}\right) s_{L}(v),
$$

with the absorbing boundary conditions

$$
\begin{aligned}
P_{L}\left(x=0^{-}, v\right) & =0, v>0, \\
P_{L}(x=d, v) & =0, v<0 .
\end{aligned}
$$

The MFPT to the boundary $E \tau_{L}$ of a particle that was injected from the left electrode is given by

$$
E_{\tau_{L}}=\int_{0}^{d} \int_{-\infty}^{\infty} \gamma P_{L}(x, v) d x d v
$$

Similarly, we define $\gamma_{P_{R}}$ as the mean time spent by a trajectory at the point $(x, v)$ prior to its absorbtion, given that it was injected to the bath from the right electrode at $x=d$ at time $t=0$. The function $P_{R}$ satisfies similar equations, and its integral is the MFPT $E_{\tau_{L}}$.

Using the definition of $f(x, v, t)$, equation (9), and changing the order of integration, we find that

$$
\begin{aligned}
\hat{f}(x, v, \theta=0) & =\int_{0}^{\infty} f(x, v, t) d t=\int_{0}^{\infty} d t \int_{0}^{t} q(s) d s \int_{t}^{\infty} p\left(x, v, t \mid t_{0}=s, \tau_{1}=r\right) p(\tau=r-s) d r \\
& =\int_{0}^{\infty} q(s) d s \int_{s}^{\infty} p(\tau=r-s) d r \int_{s}^{r} p\left(x, v, t \mid t_{0}=s, \tau_{1}=r\right) d t
\end{aligned}
$$

We identify the inner two integrals as the mean total time that a particle had spent in the $(x, v)$ location of phase space prior to its first absorbtion. Since $\int_{0}^{\infty} q(s) d s=1$, we find that

$$
\hat{f}(x, v, \theta=0)=L \gamma P_{L}(x, v)+R \gamma P_{R}(x, v),
$$


and

$$
p(x, v)=\frac{L \gamma P_{L}(x, v)+R \gamma P_{R}(x, v)}{L E \tau_{L}+R E \tau_{R}+E T},
$$

from which the concentration in phase space is given by

$$
C(x, v)=N p(x, v)=N \frac{L \gamma P_{L}(x, v)+R \gamma P_{R}(x, v)}{L E \tau_{L}+R E \tau_{R}+E T} .
$$

Equation (27) relates the probabilistic control mechanism to its resulting phase space steady state concentration, that satisfies the steady state Fokker-Planck equation with flux boundary conditions (21).

\subsection{Rate Control}

Another possible renewal control consists of two sources, placed at the left and right boundaries, which inject particles into the system. When a particle reaches the right or left boundary, its trajectory is terminated at once. The sources inject particles at identical independent distributed (i.i.d) inter-arrival random times $T_{L}$ and $T_{R}$, whose probability density functions are $f_{L}(t)$ and $f_{R}(t)$, respectively. The rates of injection are defined as

$$
\lambda_{L}=\frac{1}{E T_{L}}, \quad \lambda_{R}=\frac{1}{E T_{R}} .
$$

Note that the number of particles in the system does not remain fixed for this rate control mechanism. For any rectangle $A \subset[0, d] \times R$, we denote by $N_{A}^{L}(t)$ the number of particles in $A$ at time $t$, that were originated at the left source. Then $N_{A}^{L}(t)$ satisfies a set of renewal equations ${ }^{44}$

$$
\begin{aligned}
\operatorname{Pr}\left\{N_{A}^{L}(t)=0\right\}= & \operatorname{Pr}\{(x(t), v(t)) \notin A\} \cdot\left[\int_{0}^{t} f_{L}(s) \operatorname{Pr}\left\{N_{A}^{L}(t-s)=0\right\} d s+\int_{t}^{\infty} f_{L}(s) d s\right] . \\
\operatorname{Pr}\left\{N_{A}^{L}(t)=1\right\}= & \operatorname{Pr}\{(x(t), v(t)) \in A\} \cdot\left[\int_{0}^{t} f_{L}(s) \operatorname{Pr}\left\{N_{A}^{L}(t-s)=0\right\} d s+\int_{t}^{\infty} f_{L}(s) d s\right] \\
& +\operatorname{Pr}\{(x(t), v(t)) \notin A\} \cdot \int_{0}^{t} f_{L}(s) \operatorname{Pr}\left\{N_{A}^{L}(t-s)=1\right\} d s . \\
\operatorname{Pr}\left\{N_{A}^{L}(t)=n\right\}= & \operatorname{Pr}\{(x(t), v(t)) \in A\} \cdot \int_{0}^{t} f_{L}(s) \operatorname{Pr}\left\{N_{A}^{L}(t-s)=n-1\right\} d s \\
& +\operatorname{Pr}\left\{(x(t), v(t) \notin \notin A\} \cdot \int_{0}^{t} f_{L}(s) \operatorname{Pr}\left\{N_{A}^{L}(t-s)=n\right\} d s, \quad n>1 .\right.
\end{aligned}
$$

Thus, the expected value of $N_{A}^{L}(t)$ is given by

$$
\begin{aligned}
E N_{A}^{L}(t)= & \sum_{n=1}^{\infty} n \operatorname{Pr}\left\{N_{A}^{L}(t)=n\right\} \\
= & \operatorname{Pr}\{(x(t), v(t)) \in A\} \cdot\left[\int_{0}^{t} f_{L}(s) E N_{A}^{L}(t-s) d s+\int_{0}^{\infty} f_{L}(s) d s\right] \\
& +\operatorname{Pr}\{(x(t), v(t)) \notin A\} \cdot \int_{0}^{t} f_{L}(s) E N_{A}^{L}(t-s) d s \\
= & \operatorname{Pr}\{(x(t), v(t)) \in A\}+\int_{0}^{t} f_{L}(s) E N_{A}^{L}(t-s) d s .
\end{aligned}
$$

Dividing by the area $|A|$ of $A$ and taking the limit $|A| \rightarrow 0$, we obtain the number of particles per unit length and per unit velocity, which we call the phase space density $C^{L}(x, v, t)$. It satisfies the renewal equation

$$
C^{L}(x, v, t)=\tilde{p}_{L}(x, v, t)+\left(f_{L} * C^{L}\right)(x, v, t),
$$


Taking the Laplace transform with respect to $t$, we find that

$$
\hat{C}^{L}(x, v, \theta)=\frac{\hat{\tilde{p}}_{L}(x, v, \theta)}{1-\hat{f}_{L}(\theta)}
$$

and the steady state density is given by

$$
C^{L}(x, v)=\lim _{t \rightarrow \infty} C^{L}(x, v, t)=\lim _{\theta \rightarrow 0} \frac{\theta \hat{\tilde{p}}_{L}(x, v, \theta)}{1-\hat{f}_{L}(\theta)}=\frac{\hat{\tilde{p}}_{L}(x, v, 0)}{E T_{L}}=\lambda_{L} \hat{\tilde{p}}_{L}(x, v, 0) .
$$

We obtain from (20) that

$$
\hat{\tilde{p}}_{L}(x, v, 0)=\int_{0}^{\infty} \tilde{p}_{L}(x, v, t) d t=\gamma P_{L}(x, v) .
$$

The linearity of the expectation implies that the steady state concentration is

$$
C(x, v)=C^{L}(x, v)+C^{R}(x, v)=\gamma \lambda_{L} P_{L}(x, v)+\gamma \lambda_{R} P_{R}(x, v) .
$$

\subsection{The Renewal Control Theorem}

Even though the two control models described above are different, and have different time evolution (e.g., the number of particles inside the domain is bounded by $N$ for the former, and unbounded for the latter), they have identical steady state phase space concentrations. Indeed, choosing

$$
\lambda_{L}=\frac{N L}{L E \tau_{L}+R E \tau_{R}+E T}, \quad \lambda_{R}=\frac{N R}{L E \tau_{L}+R E \tau_{R}+E T},
$$

we find that eqs. (27) and (37) are identical. This is no mere coincidence: both controls are special cases of renewal controls.

DeFinition 3.1. A source that injects particles into the domain at random times

$0=T_{0} \leq T_{1} \leq T_{2} \ldots \leq T_{n} \leq \ldots$, such that $Y_{n}=T_{n}-T_{n-1}$ are i.i.d with $E Y_{1}<\infty$ is called a renewal source.

DEFINITION 3.2. A control made of renewal sources located at the absorbing boundary of the domain is called a renewal control.

THEOREM 3.3. The steady state phase space concentration of a renewal control is given by equation (37), where $\lambda_{L}=\frac{1}{E Y_{1}^{L}}, \lambda_{R}=\frac{1}{E Y_{1}^{R}}$ are the rates of the left and right renewal sources, respectively.

Proof. The proof is given in the previous subsection.

\subsection{Calculation of $P_{L}$ and $P_{R}$ : the albedo problem}

As seen above, all renewal control mechanisms require the knowledge of $P_{L}$ and $P_{R}$, which are the solutions of the steady state albedo problem. It was shown in Ref 39 that $P_{L}$ is given by

$$
P_{L}(x, v)=\frac{1}{\sqrt{2 \pi \epsilon}} e^{-v^{2} / 2 \epsilon} e^{-U(x) / \epsilon} Q(x, v),
$$

where $Q=Q_{B L}^{L}+Q_{B L}^{R}+Q^{O U T}$, with $Q_{B L}^{L, R}$ the boundary layer solutions, which decay exponentially fast away from the boundaries, and $Q^{O U T}$ the outer solution, given by

$$
Q^{O U T}(x, v)=C\left[\int_{0}^{x} e^{U(z) / \epsilon} d z-\frac{1}{\gamma} v e^{U(x) / \epsilon}\right]+D+O\left(\gamma^{-2}\right),
$$


with

$$
\begin{aligned}
C & =\frac{e^{U(0) / \epsilon}}{\gamma \sqrt{\epsilon}} \frac{\zeta\left(\frac{1}{2}\right)+\mathcal{B}_{0}^{L}}{\int_{0}^{d} e^{U(z) / \epsilon} d z}+O\left(\gamma^{-2}\right), \\
D & =-\frac{e^{U(0) / \epsilon}}{\gamma \sqrt{\epsilon}}\left[\zeta\left(\frac{1}{2}\right)+\mathcal{B}_{0}^{L}\right]+O\left(\gamma^{-2}\right),
\end{aligned}
$$

where $\mathcal{B}_{0}^{L}$ is a constant that depends on the velocity distribution of the left source, and $\zeta$ denotes the Riemann zeta function $\left(\zeta\left(\frac{1}{2}\right)=-1.46035 \ldots\right)$. The outer solution $Q^{O U T}$ approximates $Q$ at distances $O\left(\gamma^{-1}\right)$ away from the boundaries. Similar expressions can be written for $P_{R}$.

\subsection{Concentration Profile and Net Flux}

Equation (27) gives the concentration at $x$, which is established by the probabilistic control mechanism, as

$$
C(x)=\frac{N \gamma\left(L P_{L}(x)+R P_{R}(x)\right)}{L E \tau_{L}+R E \tau_{R}+E T}
$$

Therefore,

$$
\frac{C_{L}}{C_{R}}=\frac{L P_{L}\left(x_{1}\right)+R P_{R}\left(x_{1}\right)}{L P_{L}\left(x_{2}\right)+R P_{R}\left(x_{2}\right)} .
$$

We now solve equation (43) for the yet-undetermined parameter $L$ that keeps constant concentrations $C_{L}$ and $C_{R}$. Since $L=1-R$, the solution is given by

$$
L=\frac{C_{R} P_{R}\left(x_{1}\right)-C_{L} P_{R}\left(x_{2}\right)}{C_{L}\left[P_{L}\left(x_{2}\right)-P_{R}\left(x_{2}\right)\right]-C_{R}\left[P_{L}\left(x_{1}\right)-P_{R}\left(x_{1}\right)\right]} .
$$

Substituting in equation (42) we find that

$$
\frac{N \gamma}{E \tau+E T}=\frac{C_{L}}{L P_{L}\left(x_{1}\right)+R P_{R}\left(x_{1}\right)},
$$

and the two parameters of the control mechanism, $N$ and $L$, are uniquely determined. We assume that the left and right sources have the same velocity density distribution, $s_{L}(v)=s_{R}(-v), v>0$, which guarantees $\mathcal{B}_{0}^{L}=\mathcal{B}_{0}^{R} \equiv \mathcal{B}_{0}$. The resulting concentration at $x$ away from the boundary is given by

$$
C(x)=\frac{C_{L} e^{\left(U\left(x_{1}\right)-U(x)\right) / \epsilon} \int_{x}^{x_{2}} e^{U(z) / \epsilon} d z+C_{R} e^{\left(U\left(x_{2}\right)-U(x)\right) / \epsilon} \int_{x_{1}}^{x} e^{U(z) / \epsilon} d z}{\int_{x_{1}}^{x_{2}} e^{U(z) / \epsilon} d z}
$$

which is the same as given in eq. (3.5) of Ref 8 . Note that the constant factor $\zeta\left(\frac{1}{2}\right)+\mathcal{B}_{0}$ cancels out and therefore it cannot be seen in measuring concentrations. The total net flux is given by

$$
J(x)=N \int_{-\infty}^{\infty} v p(x, v) d v=\frac{N \gamma}{E \tau+E T} \cdot \int_{-\infty}^{\infty} v P(x, v) d v
$$

where $P=L P_{L}+R P_{R}$. The flux is constant to leading order (in $\gamma^{-1}$ ) and is given by

$$
J=\frac{\epsilon}{\gamma} \frac{C_{L} e^{U\left(x_{1}\right) / \epsilon}-C_{R} e^{U\left(x_{2}\right) / \epsilon}}{\int_{x_{1}}^{x_{2}} e^{U(z) / \epsilon} d z} .
$$

We see that the macroscopic net flux (48) is $O\left(\gamma^{-1}\right)$, and coincides with that given in eq.(3.7) of Ref 8 and in Ref 1. Theorem 3.3 then implies that eqs.(46) and (48) describe the concentration and the flux for all renewal control mechanisms. 


\section{THE NEUMANN PROBLEM}

The control mechanisms that were used in the previous sections are mathematical models of non equilibrium systems in steady state. Although these reproduce the correct concentration profile away from the boundaries, they also create spurious boundary layers. For particle systems with long range interactions, such as ions in solution, these boundary layers can distort the electrostatic field everywhere in the solution.

In this section we consider the setup described in Section 2, but with all boundaries reflecting. In other words, there are no control mechanisms involved. In the case of all reflecting boundary condition, no spurious boundary layers appear in the solution of the Fokker-Planck equation. Moreover, the Smoluchowski overdamped approximation is valid in the entire domain. In this model, time evolution is governed by the Nernst-Planck equation and no renewal operators are needed. In this setup, which describes actual channel current measurements if made with polarizing (e.g., Pt) electrodes, ${ }^{45,46}$ there is no flux in the steady state. However, the long transient is the quasi steady state that Hodgkin, Huxley, and Katz actually observed on the (biological) time scale of $1-10$ msecs.

Separation of variables in the Smoluchowski equation ${ }^{5}$

$$
\frac{\partial p_{N}(\tilde{\boldsymbol{x}}, t)}{\partial t}=-\sum_{h} \sum_{j=1}^{N_{h}} \nabla_{\boldsymbol{x}_{j}^{h}} \cdot \boldsymbol{J}_{\boldsymbol{x}_{j}^{h}}(\tilde{\boldsymbol{x}}, t)
$$

where $\boldsymbol{J}_{\boldsymbol{x}_{j}^{h}}(\tilde{\boldsymbol{x}}, t)$ is the 3 -dimensional probability flux vector of the $j$-th particle of species $h$, given by

$$
\boldsymbol{J}_{\boldsymbol{x}_{j}^{h}}(\tilde{\boldsymbol{x}}, t)=\frac{\boldsymbol{f}_{j}^{h}(\tilde{\boldsymbol{x}})}{M^{h} \gamma^{h}\left(\boldsymbol{x}_{j}^{h}\right)} p_{N}(\tilde{\boldsymbol{x}}, t)-\frac{k_{B} T}{M^{h} \gamma^{h}\left(\boldsymbol{x}_{j}^{h}\right)} \nabla_{\boldsymbol{x}_{j}^{h}} p_{N}(\tilde{\boldsymbol{x}}, t)
$$

results in

$$
p_{N}(\tilde{\boldsymbol{x}}, t)=\sum_{n=0}^{\infty} a_{n} e^{-\lambda_{n} t} \phi_{n}(\tilde{\boldsymbol{x}}),
$$

where $\lambda_{0}<\lambda_{1}<\ldots<\lambda_{n}<\ldots$ are the eigenvalues of the linear elliptic operator

$$
\sum_{h} \sum_{j=1}^{N_{h}} \nabla_{\boldsymbol{x}_{j}^{h}} \cdot \boldsymbol{J}_{\boldsymbol{x}_{j}^{h}}^{n}(\tilde{\boldsymbol{x}})=\lambda_{n} \phi_{n}(\tilde{\boldsymbol{x}})
$$

with Neumann (no-flux) boundary conditions

$$
\boldsymbol{J}_{\boldsymbol{x}_{j}^{h}}^{n}(\tilde{\boldsymbol{x}}) \cdot \nu(\tilde{\boldsymbol{x}})=0, \quad \tilde{\boldsymbol{x}} \in \partial \Omega
$$

where

$$
\boldsymbol{J}_{\boldsymbol{x}_{j}^{h}}^{n}(\tilde{\boldsymbol{x}})=\frac{\boldsymbol{f}_{j}^{h}(\tilde{\boldsymbol{x}})}{M^{h} \gamma^{h}\left(\boldsymbol{x}_{j}^{h}\right)} \phi_{n}(\tilde{\boldsymbol{x}})-\frac{k_{B} T}{M^{h} \gamma^{h}\left(\boldsymbol{x}_{j}^{h}\right)} \nabla_{\boldsymbol{x}_{j}^{h}} \phi_{n}(\tilde{\boldsymbol{x}})
$$

Clearly, the first eigenvalue is $\lambda_{0}=0$, which corresponds to the equilibrium solution $\boldsymbol{J}_{\boldsymbol{x}_{j}^{h}}^{0}(\tilde{\boldsymbol{x}})=0$ for all $\tilde{\boldsymbol{x}}, j, h$, or equivalently, $\phi_{0}(\tilde{\boldsymbol{x}})=e^{-U(\tilde{\boldsymbol{x}}) / k_{B} T}$ is the equilibrium Boltzmann distribution. This means, that the system equilibrates after sufficiently long time $\left(t \gg 1 / \lambda_{1}\right)$. As mentioned above, the long transient $\left(1 / \lambda_{2} \ll t \ll 1 / \lambda_{1}\right)$ describes measurements on the biological time scale. In this scale, a quasi steady flux flows through the channel. For such time scales, we can approximate $p_{N}(\tilde{\boldsymbol{x}}, t)$ by only the first two eigenfunctions, as the other eigenfunctions decay exponentially faster,

$$
p_{N}(\tilde{\boldsymbol{x}}, t) \approx a_{0} e^{-U(\tilde{\boldsymbol{x}}) / k_{B} T}+a_{1} \phi_{1}(\tilde{\boldsymbol{x}}) e^{-\lambda_{1} t} .
$$


Indeed, for non-interacting particles, it can be shown ${ }^{42,43}$ that the second eigenvalue $\lambda_{1}$ is much smaller than all other eigenvalues $\left(\lambda_{1} \ll \lambda_{2}\right)$, as $\lambda_{1} \propto \frac{a D}{|\Omega|}$, while $\lambda_{2} \propto \frac{D}{|\Omega|^{2 / 3}}$, where $a$ is the radius of the channel hole, $D$ is the diffusion coefficient and $|\Omega|$ is the volume of the chamber. We conjecture that $\lambda_{1} \ll \lambda_{2}$ also for the case of interacting particles. From the calculation of the eigenfunction $\phi_{1}(\tilde{\boldsymbol{x}})$, one can find the pair correlation function of the quasi steady state. The resulting pair correlation function depends on the choice of the closure relation used in the calculation, as is the case in equilibrium systems. ${ }^{47}$

\section{DISCUSSION}

The renewal controls studied here maintain systems of non interacting particles at constant average concentrations near the boundaries, and away from the boundaries they produce the stationary Nernst-Planck equation of classical diffusion theory.

We have proven that all renewal controls produce the same steady state concentration and flux, even though their time evolutions can differ qualitatively. However, renewal controls - that are widely used in computer simulations - are problematic because they produce spurious boundary layers. These boundary layers are expected to appear in interacting particle systems driven out of equilibrium by renewal controls.

The existence of such boundary layers may be of little importance if the particles interact only through short range forces, such as Lennard-Jones forces, or the forces that prevent overlap of hard spheres. However, the boundary layers can have a catastrophic effect for particles that interact through long range forces, such as ions that interact electrostatically. The net charge carried by only a tiny fraction of the total number of ions is, after all, responsible for electrical signalling in the nervous system and the electrical potentials in electrochemical cells and these potentials extend over large distances, from micron to many meters, e.g., in the neurons of whales ${ }^{48}$ as well as in inorganic applications from batteries to the trans-Atlantic cable. ${ }^{49-53}$

The boundary behavior of diffusing particles has been studied for many types of boundaries, including absorbing, reflecting, sticky boundaries, and more. ${ }^{44,54}$ In Ref 22 a sequence of Markovian jump processes is constructed such that their transition probability densities converge to the solution of the Nernst-Planck equation with given boundary conditions, including fixed concentrations and sticky boundaries.

As mentioned above, replacing the baths with renewal sources is a mathematical idealization that can produce artificial boundary effects. The renewal control effectively terminates trajectories at boundaries and starts new trajectories there. Most experiments do not. In real physical systems, particles that reach the boundary usually move into a 'guard' region, from which they often return to the domain (with some probability), with a given time distribution. To capture this behavior by a mathematical model, the entire pdf of the first passage time for the albedo problem has to be found, not only its first moment. The spurious boundary layers will be avoided if the correct time course of recycling trajectories in and out of the domain is used. We postpone this calculation, which we could not find in the literature, to a future paper.

The time evolution of systems whose average concentrations near the boundaries are maintained by renewal controls is complicated and cannot be described, in general, by a single partial differential equation. We have shown that the phase space concentration is a sum of two components, each of which satisfies a different integralpartial-differential equation with different boundary conditions. Only in the steady state does the concentration satisfy the Fokker-Planck equation with boundary conditions identical to those of the steady state albedo problem. Although the overdamped limit is a useful approximation inside the domain, it cannot be used near the boundaries, where the full Fokker-Planck equation has to be solved. For particle systems with only short range interactions, the outer solution - which is the solution to the Smoluchowski equation - determines the concentration and correlation functions away from the boundaries. One can hope that a simple boundary condition can be found for such systems, similar to the simple boundary condition that exists for non interacting systems.

A different approach to the problem is to determine the second eigenfunction of the Neumann problem for interacting particles, which describes the quasi steady state of the system, as described in Section 4 . The calculation of this eigenfunction is still an unsolved problem. The simulation of this model necessarily involves the simulation of the entire bath. This approach will be further investigated in the future. 
¿From the theoretical point of view, the absence of a rigorous mathematical theory of the boundary behavior of Brownian trajectories diffusing between fixed concentrations, based on the physical theory of the Brownian motion, is a serious gap in classical physics. This paper is a step toward the bridging of this gap.

Acknowledgment. The authors are partially supported by research grants from US-Israel Binational Science Foundation, and DARPA. ZS, AS and BN are also partially supported by research grants from the Israel Science Foundation.

\section{REFERENCES}

1. B. Hille, "Ionic channels of excitable membranes," pp. 1-814, Sinauer Associates Inc., Sunderland, 3rd ed., 2001.

2. R. S. Eisenberg, "Ionic channels in biological membranes: electrostatic analysis of a natural nano-tube," Contemp. Phys. 39, pp. 447-466, 1998.

3. R. S. Berry, S. Rice, and J. Ross, "Physical chemistry," Oxford University Press, NY, 2nd ed., 2000.

4. W. Nonner and R. S. Eisenberg, "Ion permeation and glutamate residues linked by poisson-nernst-planck theory in l-type calcium channels," Biophys. J. 75, pp. 1287-1305, 1998.

5. Z. Schuss, B. Nadler, and R. S. Eisenberg, "Derivation of pnp equations in bath and channel from a molecular model," Phys. Rev. E 64 (2-3), pp. 1-14, 2001.

6. C. Jacoboni and P. Lugli, "The monte carlo method for semiconductor device simulation," pp. 1-356, Springer Verlag, New York, 1989.

7. S. Selberherr, "Analysis and simulation of semiconductor devices," pp. 1-293, Springer Verlag, New York, 1984.

8. R. S. Eisenberg, M. M. Kłosek, and Z. Schuss, "Diffusion as a chemical reaction: Stochastic trajectories between fixed concentrations," J. Chem. Phys. 102, pp. 1767-1780, 1995.

9. H. Kramers, "Brownian motion in a field of force," Physica (Utrecht) 7, p. 284, 1940.

10. Z. Schuss, "Theory and applications of stochastic differential equations," Wiley, NY, 1980.

11. M. P. Allen and D. J. Tildesley, "Computer simulation of liquids," Oxford University Press, Oxford, 1991.

12. G. Moy, B. Corry, S. Kuyucak, and S. H. Chung, "Tests of continuum theories as models of ion channels. i. poisson-boltzmann theory versus brownian dynamics," Biophys. J. 78, pp. 2349-2363, 2000.

13. B. Corry, S. Kuyucak, and S. H. Chung, "Tests of continuum theories as models of ion channels. ii. poissonnernst-planck theory versus brownian dynamics," Biophys. J. 78, pp. 2364-2381, 2000.

14. M. Berkowitz and J. A. McCammon, "Molecular dynamics with stochastic boundary conditions," Chem. Phys. Lett. 90, pp. 215-217, 1982.

15. C. L. Brooks, III, and M. Karplus, "Deformable stochastic boundaries in molecular dynamics," J. Chem. Phys. 79, p. 6312, 1983.

16. T. Naeh, "Simulation of ionic solution," Ph.D. dissertation, Department of Mathematics, Tel-Aviv University, 2001.

17. B. Nadler, T. Naeh, and Z. Schuss, "The stationary arrival process of independent diffusers from a continuum to an absorbing boundary is poissonian," SIAM J. Appl. Math. 62, pp. 433-447, 2001.

18. B. Nadler, T. Naeh, and Z. Schuss, "Connecting a discrete ionic simulation to a continuum," SIAM J. Appl. Math. 63 (3), pp. 850-873, 2003.

19. W. Im, S. Seefeld, and B. Roux, "A grand canonical monte carlo-brownian dynamics algorithm for simulating ion channels," Biophys. J. 79, pp. 788-801, 2000.

20. W. Im and B. Roux, "Ion permeation and selectivity of ompf porin: a theoretical study based on molecular dynamics, brownian dynamics, and continuum electrodiffusion theory," J. Mol. Bio. 322 (4), pp. 851-869, 2002.

21. W. Im and B. Roux, "Ions and counterions in a biological channel: a molecular dynamics simulation of ompf porin from escherichia coli in an explicit membrane with $1 \mathrm{~m} \mathrm{kcl}$ aqueous salt solution," J. Mol. Bio. 319 (5), pp. 1177-1197, 2002. 
22. M. Schumaker, "Boundary conditions and trajectories of diffusion processes", J. Chem. Phys. , 117, pp. 2469-2473, 2002.

23. B. Corry, M. Hoyles, T. W. Allen, M. Walker, S. Kuyucak, and S. H. Chung, "Reservoir boundaries in brownian dynamics simulations of ion channels," Biophys. J. 82, pp. 1975-1984, 2002.

24. S. Wigger-Aboud, M. Saraniti, and R. S. Eisenberg, "Self-consistent particle based simulations of three dimensional ionic solutions," Nanotech 3, p. 443, 2003.

25. T. A. van der Straaten, J. Tang, R. S. Eisenberg, U. Ravaioli, and N. R. Aluru, "Three-dimensional continuum simulations of ion transport through biological ion channels: effects of charge distribution in the constriction region of porin," J. Computational Electronics 1, pp. 335-340, 2002.

26. T. A. van der Straaten, G. Kathawala, Z. Kuang, D. Boda, D. P. Chen, U. Ravaioli, R. S. Eisenberg, and D. Henderson, "Equilibrium structure of electrolyte calaculated using equilibrium monte carlo, molecular dynamics, and transport monte carlo simulation," Nanotech 3, p. 447, 2003.

27. T. A. van der Straaten, G. Kathawala, and U. Ravaioli, "Biomoca: A transport monte carlo model for ion channels," Journal of Computational Electronics 2, pp. 231-237, 2003.

28. B. Roux, "Statistical mechanical equilibrium theory of selective ion channels," Biophysical Journal $\mathbf{7 7}$, pp. 139-153, 1999.

29. A. J. Kainz and U. M. Titulaer, "The analytic structure of the stationary kinetic boundary layer for brownian particles near an absorbing wall,". Phys. A: Math. Gen. 24, pp. 4677-4695, 1991.

30. M. E. Widder and U. M. Titulaer, "The moment method for boundary layer problems in brownian motion theory," J. Stat. Phys. 56 (3/4), pp. 471-498, 1989.

31. M. E. Widder and U. M. Titulaer, "The kinetic boundary layer around an absorbing sphere and the growth of small droplets," J. Stat. Phys. 55 (5/6), pp. 1109-1128, 1989.

32. J. V. Selinger and U. M. Titulaer, "The kinetic boundary layer for the klein-kramers equation; a new numerical approach," J. Stat. Phys. 36 (3/4), pp. 293-318, 1984.

33. M. A. Burschka and U. M. Titulaer, "The kinetic boundary layer for the fokker-planck equation with absorbing boundary," J. Stat. Phys. 25, pp. 569-582, 1981.

34. U. M. Titulaer, "A systematic solution procedure for the fokker-planck equation of a brownian particle in the high-friction case," 91, pp. 321-344, 1978.

35. M. C. Wang and G. E. Uhlenbeck, "On the theory of the brownian motion ii," Rev. Mod. Phys. 17, p. 323, 1945.

36. T. W. Marshall and E. J. Watson, "A drop of ink falls from my pen ... it comes to earth, i know not when," J. Phys. A 18, pp. 3531-3559, 1985.

37. P. S. Hagan, C. R. Doering, and C. D. Levermore, "Mean exit times for particles driven by weakly colored noise," SIAM J. Appl. Math. 49 (5), pp. 1480-1513, 1989.

38. P. S. Hagan, C. R. Doering, and C. D. Levermore, "The distribution of exit times for weakly colored noise," J. Stat. Phys 54 (5/6), pp. 1321-1352, 1989.

39. M. M. Kłosek, "Half-range expansion analysis for langevin dynamics in the high-friction limit with a singular absorbing boundary condition: Noncharacteristic case," J. Stat. Phys. 79 (1/2), pp. 313-345, 1995.

40. J. M. G. Barthel and H. Krienke, "Physical chemistry of electrolyte solutions: Modern aspects," Steinkopf, Dietrich Pub., 1998.

41. W. Ebeling, R. Feistel, G. Kelbg, and R. Sandig, "Generalizations of onsager's semiphenomenological theory of electrolytic conductance," J. Non-Equilibrium Thermodynamics 3, p. 11, 1978.

42. I. V. Grigoriev, Y. A. Makhnovskii, A. M. Berezhkovskii, and V. Y. Zitserman, "Kinetics of escape through a small hole," J. Chem. Phys. 116 (22), pp. 9574-9577, 2002.

43. L. Dagdug, A. M. Berezhkovskii, S. Y. Shvartsman, and G. H. Weiss, "Equilibration in two chambers connected by a capillary," J. Chem. Phys 119 (23), pp. 12473-12478, 2003.

44. S. Karlin and H. M. Taylor, "A second course in stochastic processes," Academic Press, New York, 1981.

45. A. L. Hodgkin, A. F. Huxley, and B. Katz, "Ionic currents underlying activity in the giant axon of the squid," Arch. Sci. physiol. 3, pp. 129-150, 1949.

46. A. L. Hodgkin, A. F. Huxley, and B. Katz, "Measurement of current-voltage relations in the membrane of the giant axon of loligo," J. Physiol. (London) 116, pp. 424-448, 1952. 
47. H. T. Davis, "Statistical mechanics of phases, interfaces, and thin films (advances in interfacial engineering series)," John Wiley \& Sons, NY, 1995.

48. T. F. Weiss, "Cellular biophysics," p. 450, MIT Press, Cambridge, MA, 1996.

49. A. L. Hodgkin, "The conduction of the nervous impulse," pp. 1-108, Liverpool University Press, Liverpool, 1971.

50. W. M. Saslow, "Consider a spherical battery ...," American Journal of Physics 62, pp. 495-501, 1994.

51. W. M. Saslow, "What happens when you leave the car lights on overnight: violation of local electroneutrality in slow, steady discharge of a lead-acid cell," Physical Review Letters 76, pp. 4849-4852, 1996.

52. W. M. Saslow, "Voltaic cells for physicists: two surface pumps and an internal resistance," American Journal of Physics 67, pp. 574-583, 1999.

53. J. S. Gordon, "A thread across the ocean: The heroic story of the trans atlantic cable," p. 240, Walker, New York, 2002.

54. P. Mandl, "Analytical treatment of one-dimensional markov processes," Springer Verlag, NY, 1968. 